\title{
A Case of Congenital Bronchial Pulmonary Arterial Malformation Successfully Treated by Embolization
}

\author{
Ferdinand Shiu Kay Chu ${ }^{1}$ Ko Yung Sit ${ }^{2}$ King Ming Kwok
}

${ }^{1}$ Department of Radiology, Queen Mary Hospital, Hong Kong

${ }^{2}$ Department of Cardiothoracic surgery. Queen Mary Hospital, Hong Kong

${ }^{3}$ Tung Wah Group of Hospitals Computed Tomography Imaging Centre, Hong Kong

J Clin Interv Radiol ISVIR 2018;2:201-204

\author{
Address for correspondence Ferdinand Shiu Kay Chu, MB, BS \\ (Syd), LLB, FRCR, FACLM, FHKAM (Radiology), c/o Department of \\ Radiology, Queen Mary Hospital, Pokfulam, Hong Kong \\ (e-mail: ferd.chu@gmail.com).
}

\author{
Abstract \\ Keywords \\ - bronchial pulmonary \\ arterial malformation \\ - computed tomogra- \\ phy angiography \\ - embolization
}

Idiopathic bronchial pulmonary arterial malformation (BPAM) is a very rare condition. The authors present a case of BPAM in which a right bronchial artery communicates with a main upper lobe branch of the right pulmonary artery. It was successfully treated by embolization in one setting. The patient remained asymptomatic and well during the follow-up period. The authors therefore conclude that if the embolic material/ device is carefully chosen, it is a safe and effective means of treating BPAM.

\section{Introduction}

Bronchial pulmonary arterial malformation (BPAM) is a rare condition and only a few cases were reported in English literature. The presenting age ranges from 21 to 65 years.

\section{Case Report}

A 65-year-old, previously healthy woman presented with one episode of hemoptysis coughing up $10 \mathrm{~mL}$ of fresh blood. Chest radiograph showed a vague 2 -cm rounded opacity abutting left main bronchus ( - Fig. 1). A contrast computed tomography (CT) of the thorax and computed tomography angiography (CTA) were subsequently performed showing a cluster of abnormal serpiginous vessels in carinal region and posterior to right bronchus intermedius (-Figs. 2-4).

Angiography was performed with a view to embolization. Selective angiography showed that the right bronchial artery was tortuous and slightly hypertrophied. It widened into an aneurysm of $11 \mathrm{~mm}$ diameter before draining into the right upper lobe pulmonary arterial circulation (-Figs. 5, 6).

We further advanced selectively as far as we could go, using 2.7F Progreat Catheter (Terumo Medical Corp.) reaching beyond the aneurysmal dilatation. We deployed a $10-\mathrm{mm} \times 30-\mathrm{cm}$ Interlock coil (Boston Scientific Corp.) to form a stable "nidus" that worked as a framework to take on some further smaller coils, to form a tight cluster of coils. We deployed eight $5 \times 5.5$ $\mathrm{mm}$ and thirteen $6 \times 6.5 \mathrm{~mm}$ Vortx coils (Boston Scientific Corp.). Post-coiling angiogram showed complete obliteration of the arterial malformation (-Fig. 5). The patient did not suffer from any procedurally related complication.

The patient underwent a follow-up CTA 4 months after coil embolization. It showed that coils were in good position and that there was no sign of recurrence or further proliferation of abnormal vascular structures. The patient remained well 9 months after embolization.

\section{Discussion}

Bronchial pulmonary arterial malformations are abnormal communications between bronchial and pulmonary arteries or their branches with no obvious identifiable etiology. Yon and Ravenel ${ }^{1}$ suggested that in normal individuals, there are communications between bronchial and pulmonary circulations at arteriolar level. These communications are functionally closed or with little flow. These channels can open up or undergo neovascularization. They therefore hypothesized that BPAM arises from this anatomical basis. There have been five reported cases of idiopathic BPAM in English

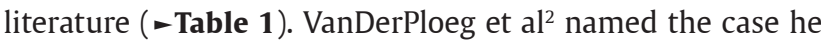
reported as pulmonary arteriovenous malformation (PAVM). The case they described had an abnormal bronchial artery
DOI https://doi.org/

$10.1055 / \mathrm{s}-0038-1676551$

ISSN 2457-0214. Radiology (c) 1 


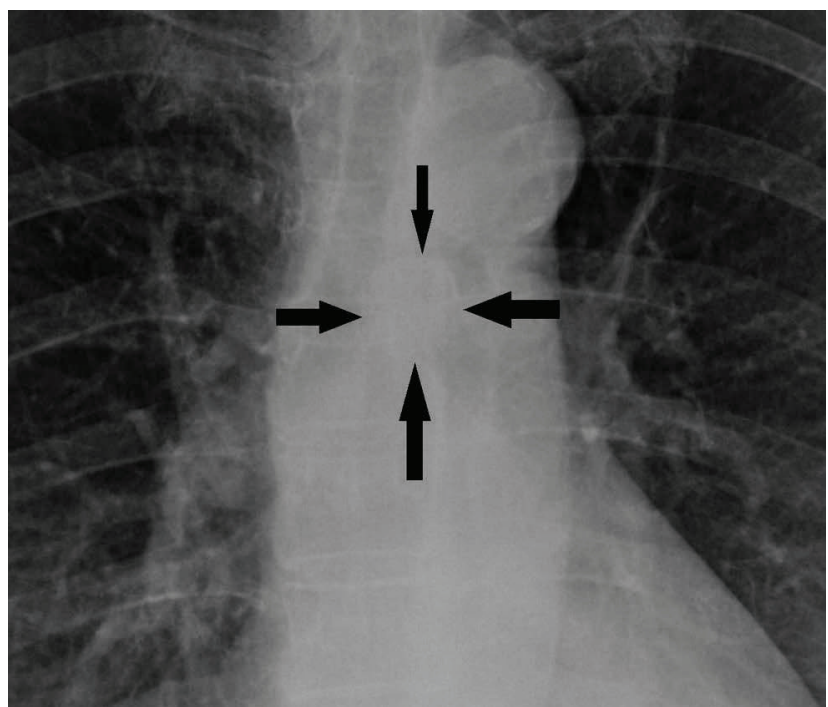

Fig. 1 Coned image of posteroanterior chest radiograph showing a $2-\mathrm{cm}$ rounded lesion (area within arrows) abutting left main bronchus.

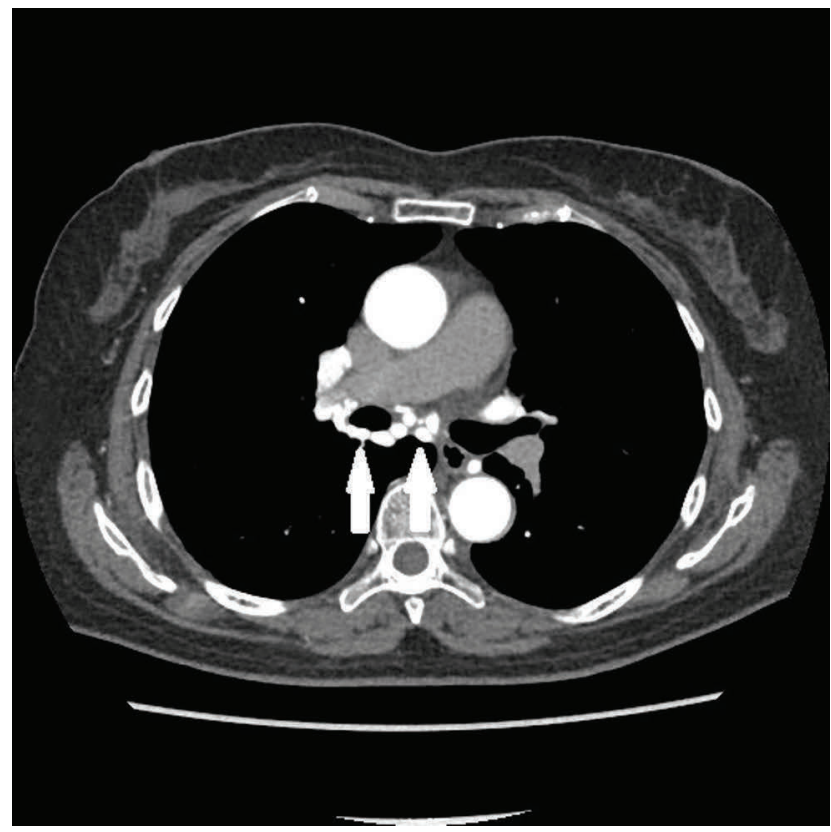

Fig. 2 The 1.25-mm thick axial images during computed tomography angiography showing the abnormal network of abnormal vessels in carinal region and behind right bronchus intermedius (arrows).

feeding an apical PAVM with outflow into right pulmonary arterial circulation. There was also abnormal communication between bronchial and pulmonary arteries; hence, it should also be considered under the same category as BPAM. Out of the five reported cases, two presented with hemoptysis, the other three presented with dyspnea. The presenting ages range from 21 to 65 years. Our patient presented with hemoptysis at the age of 65 years.

There is also another group of patients who may develop secondary abnormal communications between bronchial and pulmonary arterial circulations. Typically they are patients who have undergone Fontan operations. They are conveniently

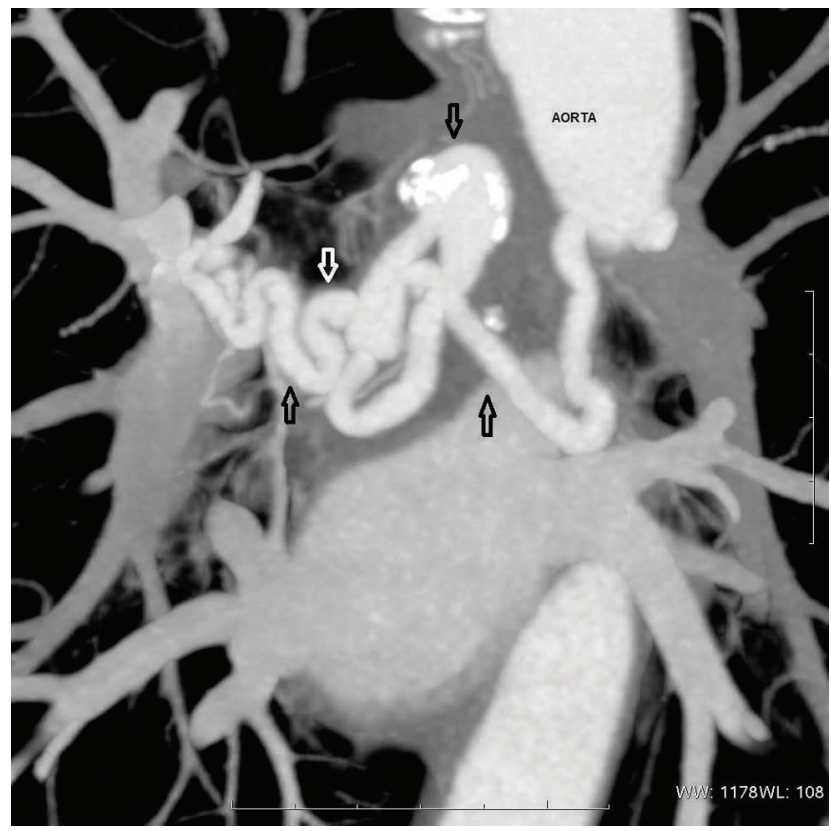

Fig. 3 Reformatted image of the computed tomography angiography scan. Open arrows denote the bronchial pulmonary arterial malformation. The segment of aorta giving rise to the abnormal bronchial artery is also labeled on the image.

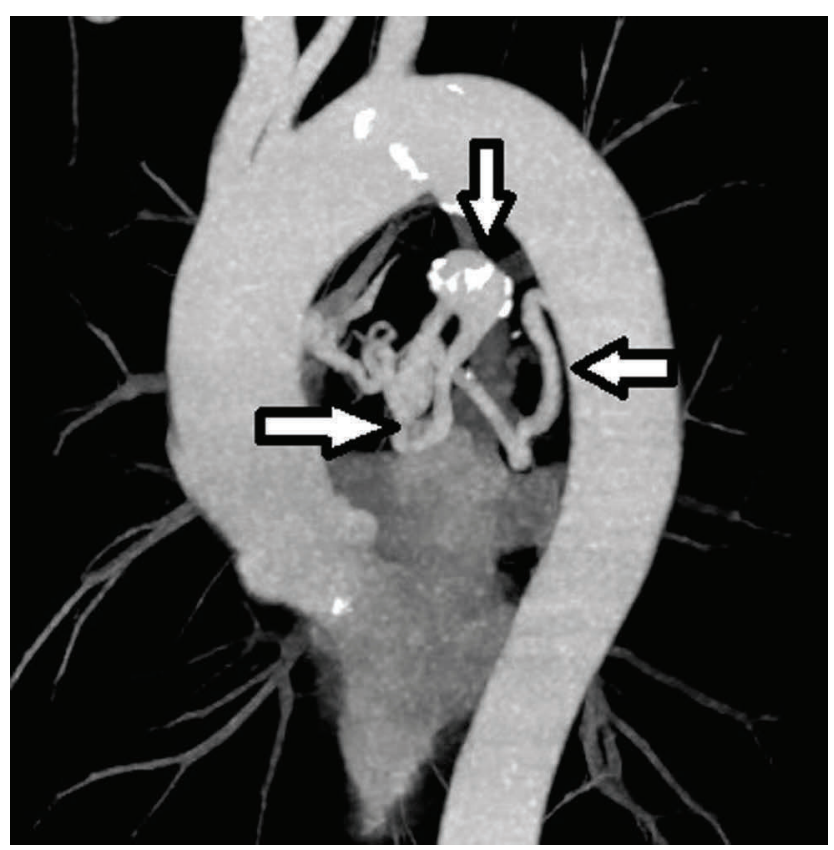

Fig. 4 Maximum intensity projection image showing the bronchial pulmonary arterial malformation (arrows).

called aortopulmonary collaterals, ${ }^{3}$ but in fact they can arise from internal mammary, subclavian, or bronchial arteries or thoracic aorta. Our patient did not have a history of previous Fontan operation and therefore does not belong to this group.

Diagnosis of BPAM on CTA alone is quite difficult. No such elaboration was found on available case reports. Contrast CT or CTA would no doubt pick up the network of abnormal blood vessels; however, it is often difficult to differentiate 


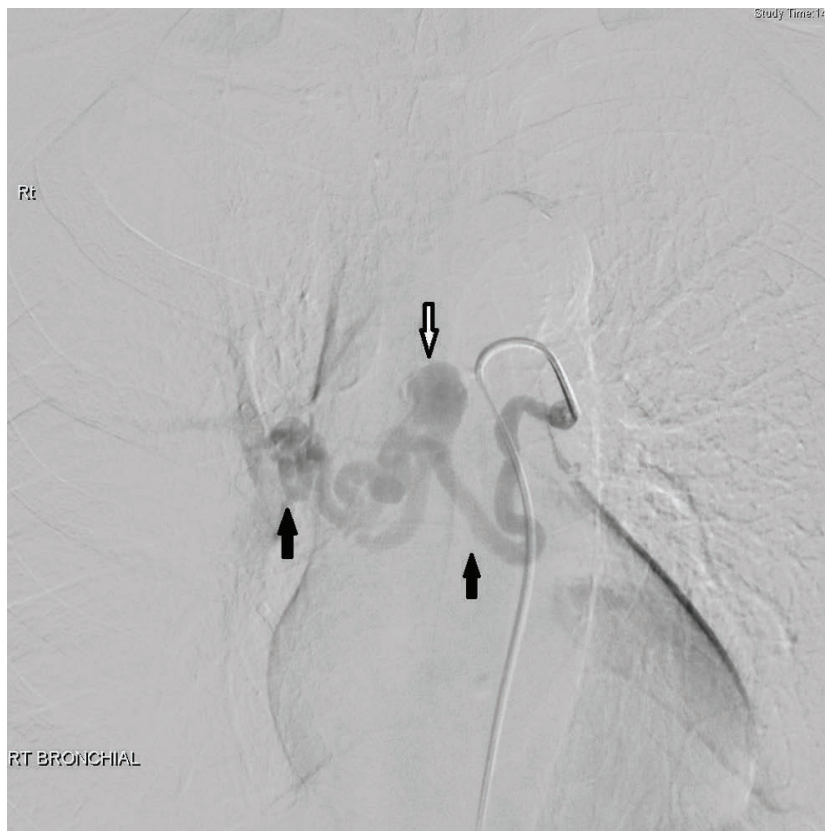

Fig. 5 Selective angiogram of right bronchial artery. The open black arrow points to the aneurysmal dilatation of the bronchial pulmonary arterial malformation. The solid black arrows point to the tortuous feeding and draining vessels on each end.

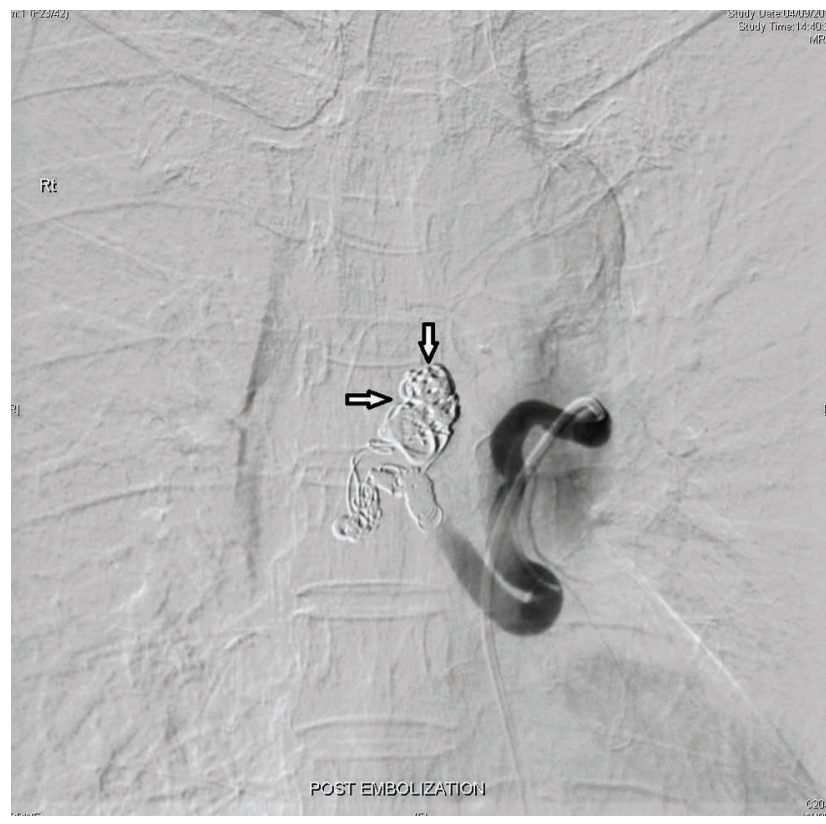

Fig. 6 Post coiling selective right bronchial arteriogram. Open arrows point to the network of coils.

Table 1 List of reported cases of BPAM or similar conditions

\begin{tabular}{|c|c|c|c|}
\hline Author & Age/sex & Presenting symptoms & Mode of treatment \\
\hline Hsieh et al $(2015)^{6}$ & $63 / \mathrm{F}$ & Exertional dyspnea & Did not specify \\
\hline $\begin{array}{l}\text { VanderPloeg et al } \\
(2014)^{2}\end{array}$ & $51 / \mathrm{M}$ & Hemoptysis & $\begin{array}{l}\text { 1. Embozene microsphere embolization } \\
\text { 2. Video-assisted thoracotomy and right upper } \\
\text { lobectomy }\end{array}$ \\
\hline $\begin{array}{l}\text { Yon and Ravenel } \\
(2010)^{1}\end{array}$ & $21 / M$ & $\begin{array}{l}\text { Shortness of breath and } \\
\text { chest pain }\end{array}$ & Coil embolization \\
\hline Kato et al (2016) & $65 / M$ & Exertional dyspnea & $\begin{array}{l}\text { Video-assisted left upper lobectomy and ligation of } \\
\text { bronchial artery aneurysm }\end{array}$ \\
\hline $\begin{array}{l}\text { Chehab et al } \\
(2014)^{4}\end{array}$ & $59 / \mathrm{M}$ & Hemoptysis & $\begin{array}{l}\text { Occlusion of right lower lobe pulmonary artery } \\
\text { using } 12 \mathrm{~mm} \text { Amplatzer vascular plug }\end{array}$ \\
\hline
\end{tabular}

Abbreviations: $\mathrm{F}$, female; $\mathrm{M}$, male.

between direct communication between bronchial artery and pulmonary artery/its branches (in the case of BPAM) versus simultaneous opacification of the abnormal vascular network and pulmonary as seen in PAVM.

Choice of embolic material depended on the anatomy and hemodynamics of the fistula. The direction of flow is from the bronchial artery (higher pressure) to the pulmonary arteries (lower pressure). The use of particle embolic material or $\mathrm{N}$-butyl cyanoacrylate glue in this fast-flowing fistula would not be suitable in our case as that would lead to nontarget embolization to the pulmonary circulation.

Yon and Ravenel ${ }^{1}$ reported the use of multiple single coils of up to $10 \mathrm{~mm}$. This would not be useful in our situation in view of the 11-mm aneurysm in our patient. These smaller coils will be unstable after deployment in our large-caliber fistula. Chehab et $\mathrm{al}^{4}$ used Amplatzer vascular plug in a secondary bronchopulmonary fistula. This would be suitable in our case, but for the fact that the tortuosity of the feeding artery in our case would preclude the deployment of such a device. VanDerPloeg et $\mathrm{al}^{2}$ and Kato et $\mathrm{al}^{5}$ reported two similar cases that were successfully treated by video-assisted surgery that is more invasive than embolization.

Our patient remained asymptomatic for 9 months up to last follow-up. The deployed coils were in stable position. We conclude that if embolic material/device is carefully chosen, embolization is an effective and safe means of treating BPAM.

\section{References}

1 Yon JR, Ravenel JG. Congenital bronchial artery-pulmonary artery fistula in an adult. J Comput Assist Tomogr 2010;34(3):418-420

2 VanDerPloeg DG, Strong WR, Krohmer SJ, O'Connor WN, Martin JT. Congenital bronchial artery to pulmonary artery fistula presenting as hemoptysis. Ann Thorac Surg 2015;99(1):e19-e20 
3 Fredenburg TB, Johnson TR, Cohen MD. The Fontan procedure: anatomy, complications, and manifestations of failure. Radiographics 2011;31(2):453-463

4 Chehab MA, Mandava S, Smillie R, Lackey AH, Chung H, Vartanian SA. Massive hemoptysis due to bronchopulmonary arterial fistula successfully treated with Amplatzer plug occlusion. J Biomed Graph Comput 2014;4(4)
5 Kato M, Morio Y, Matsunaga T, Shiraishi A, Uekusa T, Takahashi K. Bronchial-pulmonary arterial fistula with primary racemose hemangioma. Respirol Case Rep 2016;4(2):e00148

6 Hsieh C, Lee C, Fogelfeld K, Kamangar N. Bronchial artery to pulmonary artery fistula with bronchial artery aneurysm abstract SESSION TITLE: imaging student/resident case report posters. Chest 2015;148:519A 